\title{
Differentiation of Brain Waves from the Movement of the Upper and Lower Extremities of the Human Body
}

\author{
Brian Meneses-Claudio \\ Image Processing Research \\ Laboratory (INTI-Lab) \\ Universidad de Ciencias y \\ Humanidades \\ Lima, Perú
}

\author{
Witman Alvarado-Diaz \\ Student Member, IEEE \\ Image Processing Research \\ Laboratory (INTI-Lab) \\ Universidad de Ciencias y \\ Humanidades, Lima, Perú
}

\author{
Avid Roman-Gonzalez \\ Senior Member, IEEE \\ Image Processing Research \\ Laboratory (INTI-Lab) \\ Universidad de Ciencias y \\ Humanidades, Lima, Perú
}

\begin{abstract}
Currently, the study of brain waves has shown a type of alternative communication, in addition to the different applications that can be made with the brain waves obtained from each individual. The OpenBCI is an open source platform for electroencephalography (EEG), in addition to a device called Cyton Board capable of collecting brain waves and these can be sent to a computer to be processed. In this research work, a computer-machine interface is presented that may be able to collect the brain waves of individuals and process them, this is to indicate the differences between the thinking of the imaginary movement of the left and right arm, also of the leg left and right brain signals. Then, to use these brain wave differences in applications focused on people with physical disabilities.
\end{abstract}

Keywords-OpenBCI; cyton board; extremity movement; brain wave differentiation

\section{INTRODUCTION}

Currently, there are different types of communication, either between two people or more people, in order to transmit information or data and the reception. New types of communication are also created due to the current need of society such as brainwave communication for people who have different physical and / or medical problems.

Neurons communicate by means of electrical impulses can be measured by equipment capable of receiving brain waves like shows in these articles [1] [2], because they have electrodes that are located on the scalp to obtain brain signals from the individual, being an alternative communication system. Each action and / or thinks of the individual will be captured and represented in brain waves, knowing the meaning of each action.

The cerebral electrical impulses are always in constant action because we never stop thinking in other words, the brain is always working continuously, then when we imagine doing actions without moving, there is also an alteration in the brain waves.

In this research work, we will present the implementation of a brain-machine interface, for the classification of electroencephalographic signals, in this case the difference of signals between the movement of the left and right arm, in addition to the difference between the left and right leg.

The purpose of this project is to obtain the differences between the signals in order implement devices capable of acting and the reading of the electroencephalographic signals, while the individual would only have to imagine the movement of some of the extremities.

\section{Methodology}

To obtain signals from the brain, there are multiple devices, some of which have been described in [1], [2] and [3]; for the present work we will use the system provided by OpenBCI, we will use the Cyton Board in addition to the Daisy module, which together provide us with 16 channels, for obtaining data.

It is described that the communication of the Cyton Board with the PC is done through Bluetooth, so the OpenBCI Dongle is used, which provides us with the necessary communication to obtain data.

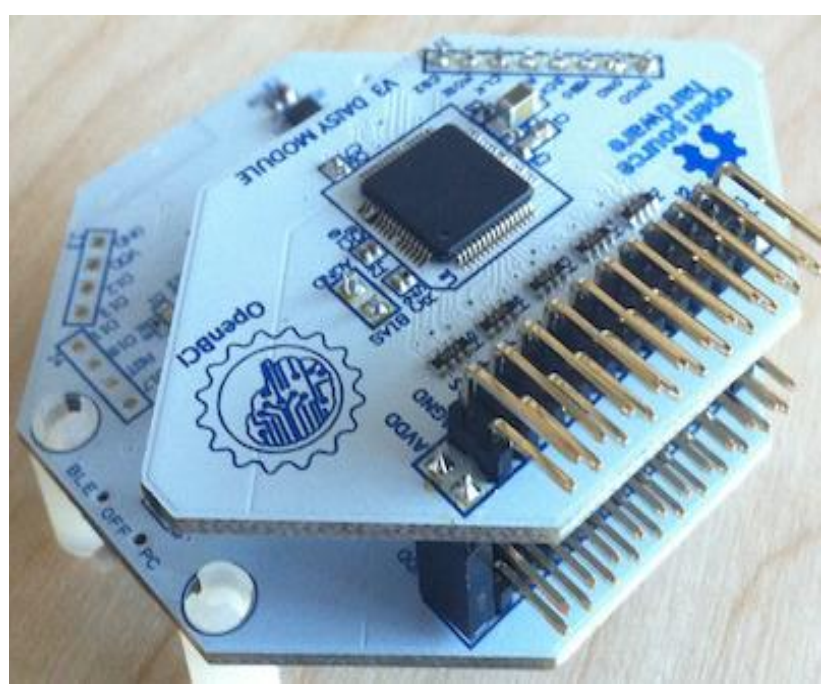

Fig. 1. Cyton Board and Daisy Module. 


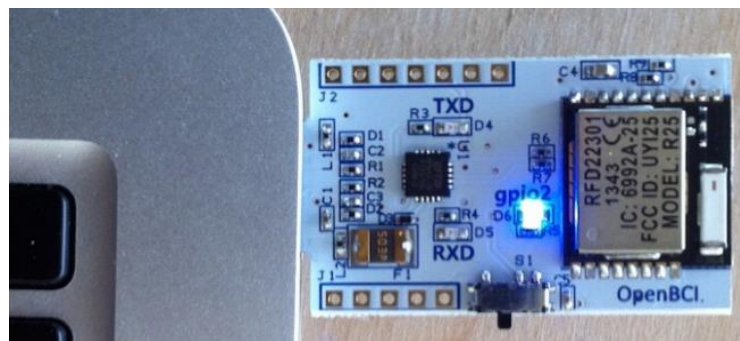

Fig. 2. OpenBCI Dongle.

For electrode placement, the international 10/20 system described in [3] is used for our case showed in figure 3, we will see the difference of the signals of the imaginary movement of the arms and legs, the electrodes will be placed in positions $\mathrm{C} 3$, $\mathrm{C} 4, \mathrm{P} 3$ and $\mathrm{P} 4$ [6] in addition to the reference connections in the ear lobes corresponding to connections A1 and A2.

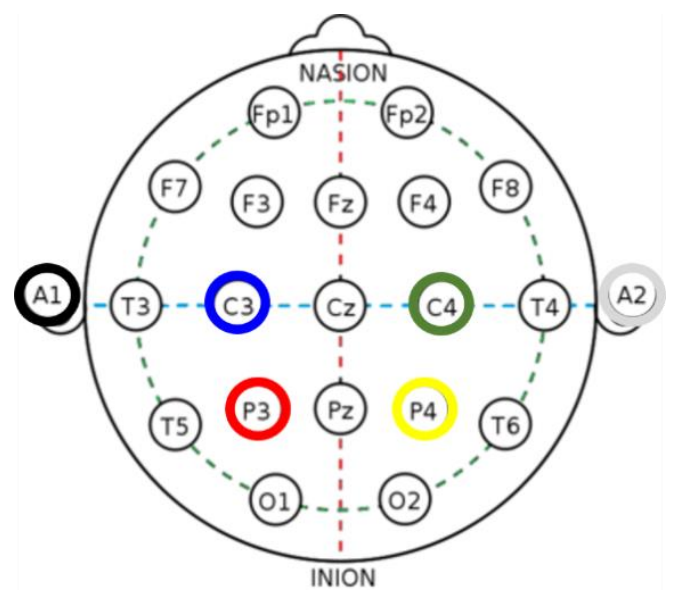

Fig. 3. Electrode Placement.

In order to improve and facilitate the placement of the electrodes in the indicated positions, the Ultracortex Mark IV system showed in figure 4 [4] was acquired, which is an opensource helmet, printable in $3 \mathrm{D}$.

For the reception of data, a graphical interface was developed, which we can control the whole process of obtaining data, described in [1], it will also be possible to control the previous processing of the data.

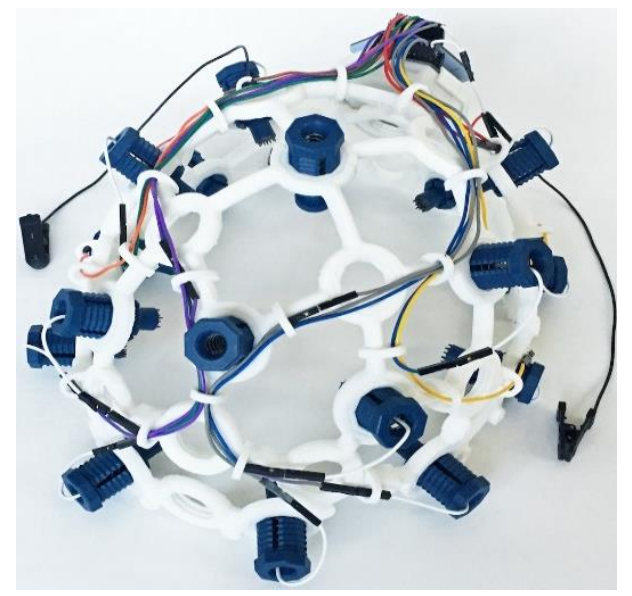

Fig. 4. Ultracortex Mark IV EEG Headset.
TABLE 1. TYPES OF BRAINWAVES

\begin{tabular}{|l|l|}
\hline \multicolumn{2}{|l|}{ BRAIN WAVES SIGNALS } \\
\hline DELTA SIGNAL & $0-3,99 \mathrm{~Hz}$ \\
\hline THETA SIGNAL & $4-7,99 \mathrm{~Hz}$ \\
\hline ALFA SIGNAL & $8-13,99 \mathrm{~Hz}$ \\
\hline BETA SIGNAL & $14-29,99 \mathrm{~Hz}$ \\
\hline GAMMA SIGNAL & $30-70 \mathrm{~Hz}$ \\
\hline
\end{tabular}

There are different types of brainwaves, each brainwave has ranges of frequency. In the table I, it will show the types of brainwaves and the frequency of each one.

Our study is based on the Brainwave type Alfa because it shows us the differences between the channel analyzed. In the results, we show the brainwave signals of the 4-channel analyzed also, to explain about the differences and indicate the imaginary movement of the upper and lower extremities.

\section{RESULTS}

\section{A. Graphic Interface}

The graphic interface has 2 main buttons: the connect button is responsible for starting Python and the necessary libraries; the start button is responsible for starting the data capture with the specified parameters; in addition, the reader has three secondary buttons that are the Python button, responsible for starting only Python; the BCILAB button responsible for starting only the libraries, in addition to the analyze button, which will result in 4 graphs in which we can buy the signals obtained.

The graphic interface has an area where the user must enter basic information such as: name, repetitions, time and rest; repetitions are the number of times the system will capture data for each patient, as well as the number of different files that it will generate; in the request of time the time in which the person, to whom the data is being recorded, must remain, must be concentrated in the indications given; at rest you must enter the rest time you will have, the person to whom, are taking the data, by default it will be 3 seconds between one repetition and another.

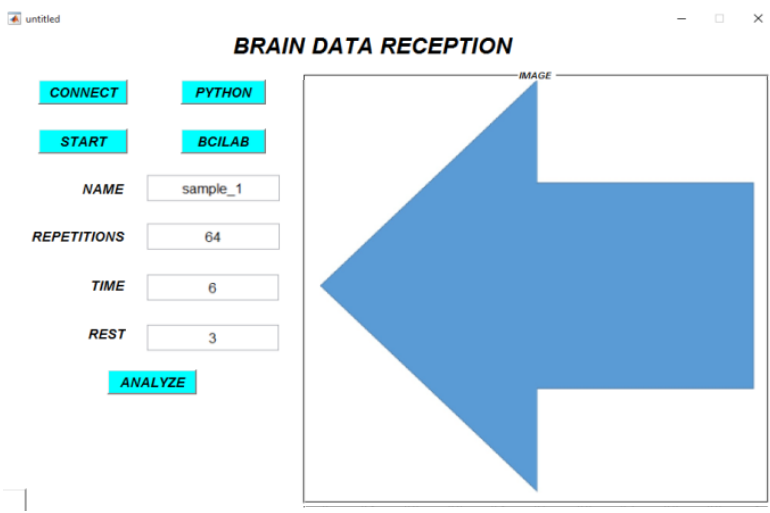

Fig. 5. Graphic Interface. 
An area was placed where the respective indications will be shown for the people to whom the data is being taken, this area shows random images, which correspond to the four most known directions (right, left, forward and backward).

Finally, the user interface is as the following Figure 5, in which we can see all the elements described above.

This stage of the project is the most important because it is where all the necessary processing for the operation of the system will be carried out. Before processing, it is necessary to capture data, for which it takes 9 seconds, 6 data and 3 rest, repeating 64 times the process, this capture was made to 10 different people, being 6 women and 4 men, as Test phase, they are people with no physical disabilities, as a first study, we want to know about what they are imaging and then it will be use for people with physical disabilities.

In order to better observe the signals, 2 filters were applied, a filter with a pass band 5 to $15 \mathrm{~Hz}$ and a band reject filter to eliminate the $60 \mathrm{~Hz}$ noise [7], was implemented with the following code:

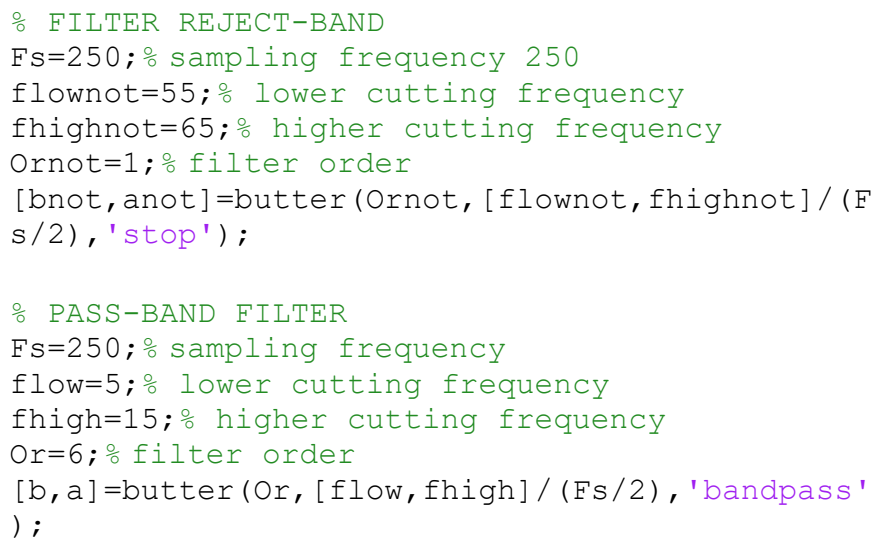

The Fast Fourier Transform FFT was found and all the signals corresponding to a single direction were averaged resulting in 4 comparative signals for each participant, in which it is shown that it is possible to differentiate the signals for the orders that were shown in the graphical interface.

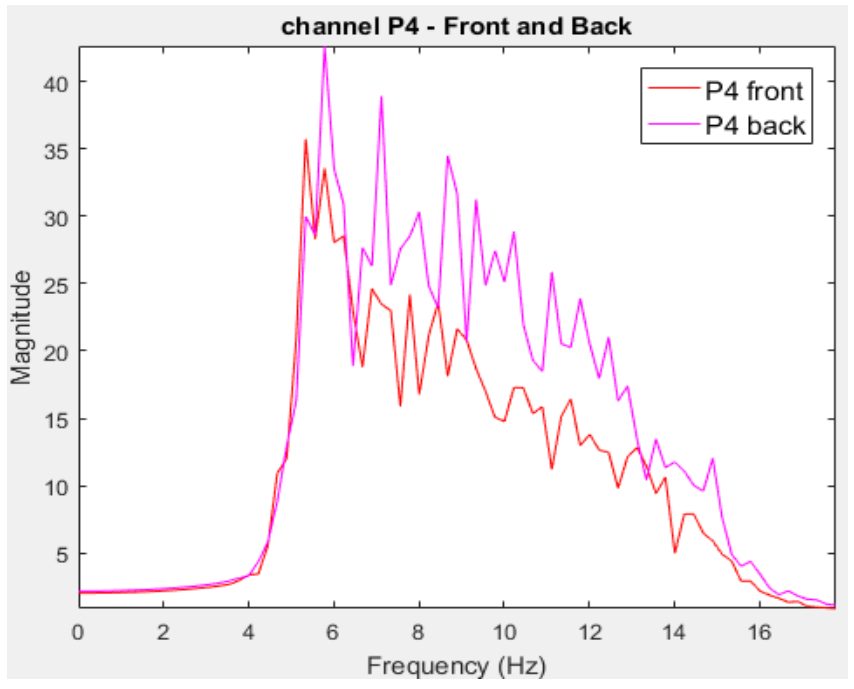

Fig. 6. Comparison in the Position P4.
In the figure 6 and 7, it will show the brainwaves in the channel P3 and P4, these signals are the imaginary movement of the left and right legs.

In the following image, we can observe the clear differentiation that exists between the two signals corresponding to two different directions, for the same electrode, which was placed in position $\mathrm{P} 4$ of the international system 10/20 [5]; it can mainly be observed that the most notable differentiation is between approximately 8 and $13 \mathrm{~Hz}$, and these values correspond to the frequency range of the alpha waves.

As in the previous image, the comparison is also made in position $\mathrm{P} 3$, in which we also see that there is a clear differentiation of the signals. It should be noted that the signals that are within the range $8-13 \mathrm{~Hz}$ are the most relevant for the study [6].

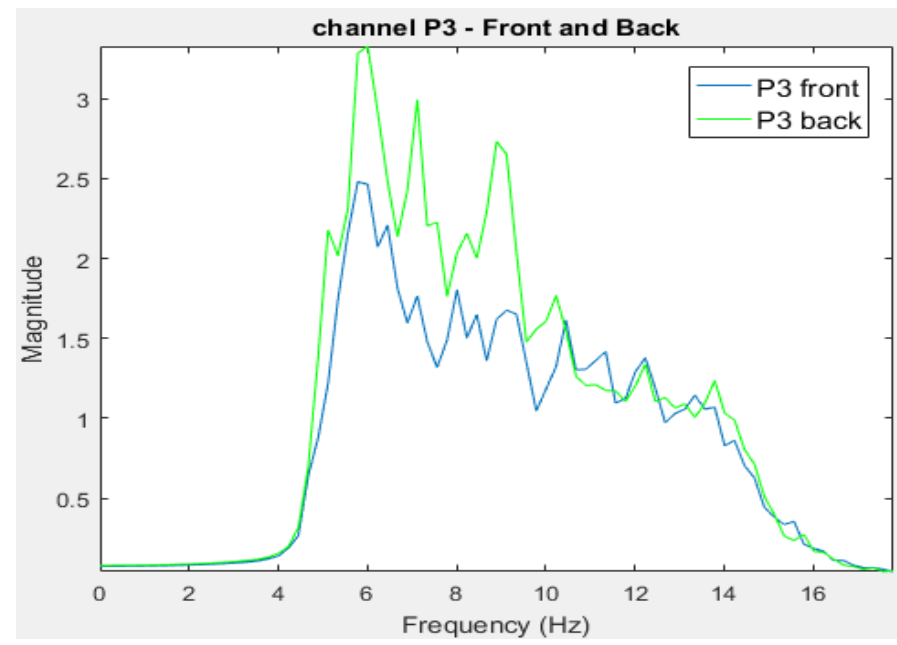

Fig. 7. Comparison in the Position P3.

In the figure 8 and 9, it will show the brainwaves in channel $\mathrm{C} 3$ and $\mathrm{C} 4$, these signals are the imaginary movement of the left and right arms.

Additionally, we analyzed the signals coming from the imaginary movement of the arms, one of which we can see the next image, which corresponds to the position $\mathrm{C} 4$.

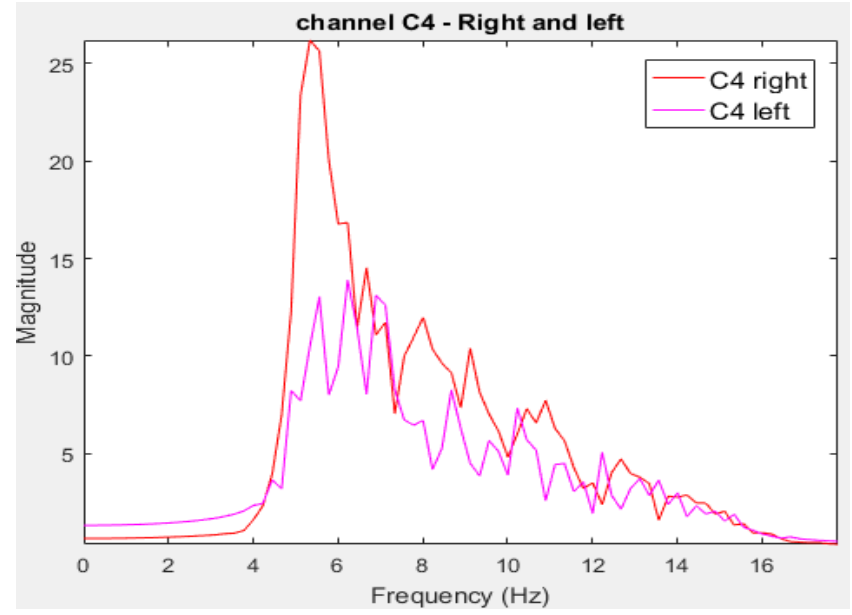

Fig. 8. Comparison in the Position C4. 


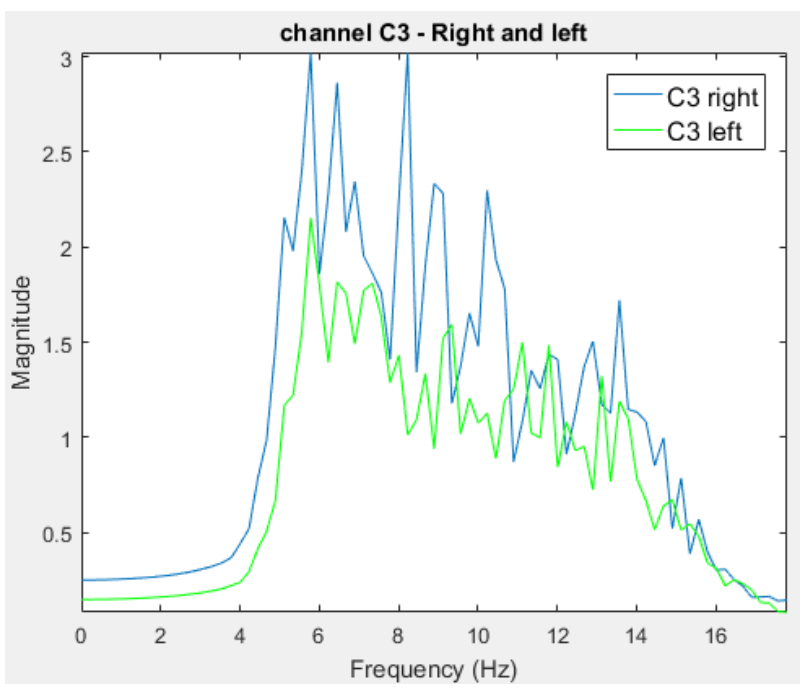

Fig. 9. Comparison in the Position C3.

Finally, we compare the signals in position C3, where we also see that it is possible to achieve a differentiation of the signals.

With the analysis of the signals, we can realize that it is possible to achieve the differentiation of the same with 4 different instructions, which were given as follows:

Arrow up = imaginary movement of the Right leg.

Arrow Backward = imaginary movement of the Left leg.

Arrow to the right = imaginary movement of the Right arm.

Arrow to the left = imaginary movement of the Left arm.

This shows that in the future, we will be able to apply differentiation algorithms of the signals, in order to control real-world objects.

Mainly the help is sought for people with physical disabilities that require electronic systems capable of actions based on the person's thinking [8] [9].

\section{DISCUSSION}

The present work confirms the processing of brain waves for the identification of the differentiation of thoughts of the movement of the upper and lower extremities, besides identifying the difference also indicate that, obtaining these results, they can be applied to electronic equipment to act at the moment of identifying the differentiation.

Currently, there are several studies that study with brain waves for processing and then their study, in this case shows the difference that exists in the imagination of the movement of the lower upper extremities.

The study of communication by brain waves is an innovative technology because it can improve the quality of life of people who have physical and / or motor disabilities.

As a future work, we are going to use these data in electronic devices for people who have physical disabilities because they have just their brain as a method of communication, so using these data, we can build a wheelchair moving by their brainwaves, it will work just imaging the movement of the upper and lower extremities.

\section{CONCLUSIONS}

It is concluded that, knowing the differentiation of the cerebral waves of the movement of the upper and lower extremities, electronic devices can be constructed that can work only with the action of the thought of the movements.

It is concluded that the application of an electro conductive gel was used in order to facilitate obtaining data from the electrodes, as well as to more easily identify the differentiation of brain waves.

It is concluded that the individual must be on alert because, during the analysis, the study can be somewhat ambiguous, being a bit boring for the individual. For this reason, alertness is required, in addition, to do well the tasks indicated.

The differences from the brainwaves showed in this paper are remarkable, also these data are digital so a microcontroller could use them to move electronic devices and help people with motor disabilities.

As a future work, theses data are going to be useful to make complete system for follow the instructions showed by the brain imagining movement for people with motor disabilities. Also, a wheelchair is going to be implemented and using the brainwaves, people with motor disabilities can move by themselves just imaging movement for their arms and legs.

\section{REFERENCES}

[1] W. Alvarado-Díaz, B. Meneses-Claudio, and A. Roman-Gonzalez, "Implementation of a brain-machine interface for controlling a wheelchair," in 2017 CHILEAN Conference on Electrical, Electronics Engineering, Information and Communication Technologies, CHILECON 2017 - Proceedings, 2017.

[2] B. Meneses-Claudio, W. Alvarado-Diaz, A. Roman-Gonzalez, and E. Z. Villaorduña, "Implementation of a Wireless system for the processing and comparison of cerebral waves of patients with Amyotrophic Lateral Sclerosis through matlab identifying their basic needs," in 2017 CHILEAN Conference on Electrical, Electronics Engineering, Information and Communication Technologies, CHILECON 2017 Proceedings, 2017.

[3] A. Roman-Gonzalez, "Communication Technologies Based on Brain Activity," pp. 91-96, 2012.

[4] OpenBCI, "Ultracortex Mark IV | Documentation," 2016. [Online]. Available: http://docs.openbci.com/Headware/01-Ultracortex-Mark-IV. [Accessed: 31-Jul-2018].

[5] Alcoba, S., Minguillón, J., Morillas, C., Carrillo, R., Pelayo, F., \& López-Gordo, M. Á. "Plataforma inalámbrica configurable para BCI". 7o Simp. CEA Bioingeniería, Málaga, 27-32, 2015.

[6] Rodríguez, V., Jahel, G., Cárdenas, U., \& Fernando, D.. Implementación de un Sistema BCI para el Análisis del Comportamiento de Bioseñales Neurológicas" (Bachelor's thesis, Universidad del Azuay), 2017.

[7] Correa Oliva, C. J. "Adquisición y procesamiento multisensorial en FPGA para EEG", 2017.

[8] Iglesias López, P. "Brain-Computer Interfaces: Desarrollo de un sistema de identificación de estados mentales alfa", 2016.

[9] Jiménez Guevara, M. P. "Sistema electrónico de iluminación (ON-OFF) mediante el control de señales cerebrales basado en tecnología EEG" (Bachelor's thesis), 2017. 